\title{
The vector and axial currents in Wilson chiral perturbation theory
}

\section{Sinya Aoki}

Graduate School of Pure and Applied Sciences, University of Tsukuba, Tsukuba, Ibaraki 305-8571, Japan

E-mail: saoki@het.ph.tsukuba.ac.jp

\section{Oliver Bär}

Institute of Physics, Humboldt Universität zu Berlin, Newtonstraße 15, 12489 Berlin, Germany

Email: obaerephysik.hu-berlin.de

\section{Stephen R. Sharpe*}

Physics Department University of Washington Seattle, WA 98195-1560

E-mail: sharpe@phys.washington.edu

\begin{abstract}
We reconsider the construction and matching of the vector and axial currents in Wilson Chiral Perturbation Theory (WChPT), the low-energy effective theory for lattice QCD with Wilson fermions. In particular, we discuss in detail the impact of the finite renormalization of the currents on their matching from the lattice theory to WChPT. We explicitly show that imposing chiral Ward-Takahashi identities on the currents leads, in general, to additional terms of $\mathrm{O}(a)$ in the axial current. We illustrate the impact on physical quantities by computing the pion decay constant to one-loop order in the two flavor theory. Our result differs from previously published ones.
\end{abstract}

The XXVII International Symposium on Lattice Field Theory

July 26-31, 2009

Peking University, Beijing, China

\footnotetext{
* Speaker.
} 


\section{Introduction}

Lattice QCD breaks several global symmetries of the (formal) continuum theory. It follows that the corresponding lattice currents are not exactly conserved, and require finite renormalization to match to continuum currents. A standard method for determining the renormalization factors is to impose Ward-Takahashi identities (WTI) which would hold were the symmetry exact. The resulting renormalization factors depend on the WTI chosen, although this dependence vanishes with some power of the lattice spacing.

An important example is the breaking of chiral symmetry when using Wilson fermions (possibly improved). The current is then the non-singlet axial current, and one determines $Z_{\mathrm{A}}$ by imposing a WTI following from $S U(2)_{L} \times S U(2)_{R}$ chiral symmetry (assuming two flavors) [1].

In this note we address the question of how $Z_{\mathrm{A}}$ depends upon the choice of WTI, and how the dependence enters into results for matrix elements of the renormalized axial current, e.g. $f_{\pi}$. As noted already, the variation in $Z_{\mathrm{A}}$ is a discretization error, and thus the question is naturally addressed using Wilson chiral perturbation theory (WChPT), i.e. ChPT with discretization errors incorporated [2]. It turns out, however, that the results on this issue in the literature [3,4] are incorrect. Here we present a brief summary of our recent reanalysis [5], explaining what was missed in the earlier work, and commenting on the significance of our new results. Our analysis controls only discretization errors linear in $a$, and thus is useful only if either the action or the currents (or both) are unimproved. The methodology can, however, be extended to higher order in $a$, and the overall conclusions are applicable more generally.

We also discuss, in tandem, the corresponding issue for ultra-local lattice vector currents. Here there is an exactly conserved lattice current, but it is not ultra-local, and often it is computationally simpler to use the ultra-local version. Since the latter current is not conserved, it receives a finite renormalization, one that can again be determined by imposing an appropriate WTI.

\section{Currents and Renormalization Conditions}

We consider lattice QCD with $N_{f}=2$ Wilson fermions with lattice spacing $a$. The ultra-local flavor non-singlet vector and axial currents are related to renormalized currents as follows:

$$
\begin{aligned}
V_{\mu, \text { ren }}^{b} & =Z_{\mathrm{V}} V_{\mu, \mathrm{Loc}}^{b} & & V_{\mu, \mathrm{Loc}}^{b}(x)=\bar{\psi}(x) \gamma_{\mu} T^{b} \psi(x) \\
A_{\mu, \text { ren }}^{b} & =Z_{\mathrm{A}} A_{\mu, \mathrm{Loc}}^{b} & & A_{\mu, \mathrm{Loc}}^{b}(x)=\bar{\psi}(x) \gamma_{\mu} \gamma_{5} T^{b} \psi(x)
\end{aligned}
$$

The $Z$-factors depend on the action as well as on the WTI used to fix them. For simplicity, we work here and below in the massless limit, attained by sending $\kappa \rightarrow \kappa_{c}$. Since we work only at linear order in $a$, we do not encounter the possible Aoki-phase or first-order transition at small masses induced by discretization errors of $O\left(a^{2}\right)$ [2]. This simplification also means that we drop all terms proportional to $a m .^{1}$

The specific WTI that we use to determine the $Z$-factors are as follows. For the vector current, we impose that the pion have the correct "charge":

$$
\left\langle\pi^{b}(\vec{p})\left|V_{0, \text { ren }}^{c}\right| \pi^{d}(\vec{p})\right\rangle=\varepsilon^{b c d} 2 E
$$

\footnotetext{
${ }^{1}$ In practice, attaining zero quark mass requires an extrapolation, or the use of Schrödinger-functional boundary conditions as an infrared regulator.
} 
One must have that $\vec{p} \neq 0$ in order that $E \neq 0$ (since we are in the massless limit). The renormalization factor will, in general, depend on $\vec{p}$.

For the axial current we impose " $\delta_{A} A \sim V$ " between pion states [1]

$$
\begin{aligned}
\int \mathrm{d} \vec{x} \varepsilon^{a b c} \varepsilon^{c d e}\left\langle\pi^{d}(\vec{p})\right|\left[A_{0, \text { ren }}^{a}\left(y_{0}+t, \vec{x}\right)\right. & \left.-A_{0, \text { ren }}^{a}\left(y_{0}-t, \vec{x}\right)\right] A_{0, \text { ren }}^{b}(y)\left|\pi^{e}(\vec{q})\right\rangle \\
& =2 i \varepsilon^{c d e}\left\langle\pi^{d}(\vec{p})\left|V_{0, \text { ren }}^{c}(y)\right| \pi^{e}(\vec{q})\right\rangle .
\end{aligned}
$$

Here we need either $\vec{p}$ or $\vec{q}$ (or both) to be non-vanishing for the right-hand-side to be non-zero, and we choose to take both non-vanishing to avoid infrared divergences. Once we have a renormalized vector current (which enters on the right-hand-side) we can use this WTI to determine $\left|Z_{\mathrm{A}}\right|$. Here the result can depend not only on the pion momenta but also on the Euclidean time separating the two axial currents (which traces back to the size of the region over which the axial rotation is applied).

Other renormalization conditions are sometimes used in practice, but we have chosen these two examples as they can be analyzed using ChPT. We also note that, in practice, one cannot consider many choices for the external pion momenta and time separations, due to the associated computational cost and the degradation of the signal as $|\vec{p}|$ increases. In contrast, ChPT allows one to study all momenta in the chiral regime.

\section{Mapping Currents into the Effective Chiral Theory}

The required calculation is now clear: map the lattice currents into the effective chiral theory and then evaluate the matrix elements appearing in the WTI (2.3) and (2.4). The mapping is done, following [2], in two steps, first from the lattice into the Symanzik effective continuum theory, and from there into the chiral effective theory. Since $Z_{\mathrm{V}}$ and $Z_{\mathrm{A}}$ are overall constants to be determined at the end, the mapping needs to be done for the bare lattice currents.

In the first step, using the symmetries of the lattice theory, one finds [1]:

$$
\begin{aligned}
& V_{\mu, \mathrm{Loc}}^{b} \simeq V_{\mu, \mathrm{Sym}, \mathrm{Loc}}^{b}=\frac{1}{Z_{\mathrm{V}}^{0}}\left(V_{\mu, \mathrm{ct}}^{b}+a \bar{c}_{\mathrm{V}} \partial_{\nu} T_{\mu \nu, \mathrm{ct}}^{b}\right)+\mathrm{O}\left(a^{2}\right) \\
& A_{\mu, \mathrm{Loc}}^{b} \simeq A_{\mu, \mathrm{Sym}, \mathrm{Loc}}^{b}=\frac{1}{Z_{\mathrm{A}}^{0}}\left(A_{\mu, \mathrm{ct}}^{b}+a \bar{c}_{\mathrm{A}} \partial_{\mu} P_{\mathrm{ct}}^{b}\right)+\mathrm{O}\left(a^{2}\right)
\end{aligned}
$$

where the continuum bilinears $\mathscr{O}_{\text {ct }}$ take their usual forms. This result displays all power-law dependence on $a$ explicitly (here at linear order); a logarithmic dependence still enters through the implicit dependence of $\bar{c}_{\mathrm{V}, \mathrm{A}}$ and $Z_{\mathrm{V}, \mathrm{A}}^{0}$ on $g(a)$. Z -factors are needed in these mappings because the currents $V_{\mu, \mathrm{ct}}^{a}$ and $A_{\mu, \mathrm{ct}}^{a}$ are conserved while the lattice currents are not. The superscript indicates that the $Z_{\mathrm{V}, \mathrm{A}}^{0}$, while containing perturbative contributions to all orders, do not include $O(a)$ terms. In practice, we know $Z_{\mathrm{V}, \mathrm{A}}^{0}$ only approximately, but this does not matter since, as will be seen below, they cancel once we normalize the currents non-perturbatively.

The mapping of the currents in (3.1-3.2) into Wilson ChPT is more subtle. One cannot simply take the WChPT action (including $O(a)$ terms) and determine the currents using the Noether procedure, as done in Ref. [3], because the symmetries are broken explicitly. Instead, we have used the following two methods. 
- Following continuum ChPT, construct the generating functional, including lattice artefacts using standard spurion methods, and obtain the currents by taking derivatives with respect to sources. One must treat the $O(1)$ and $O(a)$ parts of the currents (3.1-3.2) separately.

- Write down the most general currents compatible with the symmetries, and impose appropriate WTI by hand.

The first approach was used in Ref. [4], and more fully justified in [6]. We have checked the analysis using a more general method. The second approach is more direct, and we used it to check that no subtleties had been overlooked in the first method. We find that both approaches agree.

The resulting mappings are (in agreement with the results of Ref. [4], and dropping here and henceforth a ubiquitous " $+O\left(a^{2}\right)$ "):

$$
\begin{gathered}
V_{\mu, \mathrm{ct}}^{b}+a \bar{c}_{\mathrm{V}} \partial_{\nu} T_{\mu v, \mathrm{ct}}^{b} \simeq V_{\mu, \mathrm{eff}}^{b}=V_{\mu, \mathrm{LO}}^{b}\left(1+\frac{4}{f^{2}} \hat{a} W_{45} \bar{c}_{\mathrm{SW}}\left\langle\Sigma+\Sigma^{\dagger}\right\rangle\right), \\
A_{\mu, \mathrm{ct}}^{b}+a \bar{c}_{\mathrm{A}} \partial_{\mu} P_{\mathrm{ct}}^{b} \simeq A_{\mu, \mathrm{eff}}^{b}=A_{\mu, \mathrm{LO}}^{b}\left(1+\frac{4}{f^{2}} \hat{a} W_{45} \bar{c}_{\mathrm{SW}}\left\langle\Sigma+\Sigma^{\dagger}\right\rangle\right)+4 \hat{a} W_{\mathrm{A}} \bar{c}_{\mathrm{A}} \partial_{\mu}\left\langle T^{b}\left(\Sigma-\Sigma^{\dagger}\right)\right\rangle,
\end{gathered}
$$

where $V_{\mu, \mathrm{LO}}^{a}$ and $A_{\mu, \mathrm{LO}}^{a}$ are the standard, leading-order ChPT currents, $\Sigma$ contains the pion fields in the standard way, $\hat{a}=2 W_{0} a$, and the $W_{X}$ are unknown low energy coefficients (LECs) associated with the lattice artifacts. The coupling $\bar{c}_{\mathrm{SW}}$ is the coefficient of the "clover-term" in the Symanzik action, and, like $\bar{c}_{\mathrm{V}, \mathrm{A}}$, is only known approximately. This is now seen to be unimportant, however, since it multiplies an unknown LEC. Note that for the vector current the $\bar{c}_{\mathrm{V}}$ term in the Symanziktheory current does not lead to an $O(a)$ contribution to the current in WChPT, while the $\bar{c}_{\mathrm{A}}$ term in the axial current does.

Putting in the overall factors, the renormalized ultra-local vector and axial currents map into WChPT as

$$
V_{\mu, \text { ren }}^{b} \simeq \frac{Z_{\mathrm{V}}}{Z_{\mathrm{V}}^{0}} V_{\mu, \text { eff }}^{b}, \quad A_{\mu, \text { ren }}^{b} \simeq \frac{Z_{\mathrm{A}}}{Z_{\mathrm{A}}^{0}} A_{\mu, \text { eff }}^{b} .
$$

In Ref. [4], the factors of $Z_{\mathrm{V}} / Z_{\mathrm{V}}^{0}$ and $Z_{\mathrm{A}} / Z_{\mathrm{A}}^{0}$ were set to unity, based on an erroneous argument. In fact, these factors can differ from unity at $\mathrm{O}(a)$, as will be seen shortly.

\section{Determining the Renormalization Factors}

We now use the mapped currents of (3.5) to evaluate the WTI in WChPT. We work in the power-counting in which $a \sim p^{2}$, so that a tree-level calculation suffices to obtain the terms linear in $a$. Evaluating the WTI (2.3) we find the simple result

$$
Z_{\mathrm{V}}=Z_{\mathrm{V}}^{0} \quad V_{\mu, \text { ren }}^{b}=V_{\mu, \text { eff }}^{b} .
$$

Hence, no additional $\mathrm{O}(a)$ terms are introduced by the current renormalization. We discuss why this is in Ref. [5]; see also Ref. [4].

For the axial current, the WTI (2.4) gives, after some calculation,

$$
\frac{Z_{\mathrm{A}}}{Z_{\mathrm{A}}^{0}}=1-\frac{4 \hat{a}}{f^{2}}\left(W_{45} \bar{c}_{\mathrm{SW}}+W_{\mathrm{A}} \bar{c}_{\mathrm{A}}\right) z_{\mathrm{A}}(t), \quad z_{\mathrm{A}}(t)=1-\cosh [t(|\vec{p}|-|\vec{q}|)] \exp [-|t||\vec{p}-\vec{q}|] .
$$


In contrast to the vector current we do find a non-vanishing correction of $\mathrm{O}(a)$. This correction depends on the separation $t$ between the axial currents and upon the external states, as expected. Thus, the renormalized ultra-local axial current maps into WChPT as

$$
A_{\mu, \text { ren }}^{b} \simeq\left[1-\frac{4 \hat{a}}{f^{2}}\left(W_{45} \bar{c}_{\mathrm{SW}}+W_{\mathrm{A}} \bar{c}_{\mathrm{A}}\right) z_{\mathrm{A}}(t)\right] A_{\mu, \mathrm{eff}}^{b} .
$$

This is our major new result.

We conclude that the $a$ dependence of $A_{\mu \text {,eff }}^{b}$, derived using symmetries, is supplemented by an additional discretization error resulting from the application of the normalization condition at non-zero $a$. There are three distinct cases (recalling that $\vec{p}, \vec{q} \neq 0$ ):

1. $\vec{p}=\vec{q}$. This is the simplest case to implement practically, and leads to $z_{\mathrm{A}}(t)=0$. Thus it turns out that, in this case, there are no additional $O(a)$ terms introduced by the current normalization.

2. $\vec{p}$ parallel to $\vec{q}$. Then, for $|t| \gg 1 /|\vec{p}-\vec{q}|$, the product of cosh and exponential becomes $1 / 2$, and so $z_{\mathrm{A}} \rightarrow 1 / 2$.

3. All other non-vanishing $\vec{p}$ and $\vec{q}$. Here, for $|t| \gg 1 /|\vec{p}-\vec{q}|$, the exponential overwhelms the cosh and $z_{\mathrm{A}} \rightarrow 1$.

We stress that in both the second and third cases $z_{\mathrm{A}}$ depends on $t$ for non-asymptotic values of $t$. In any case, our main point is that the implementation of the renormalization condition leads, in general, to a non-trivial $\mathrm{O}(a)$ correction to the current. This is what was missed in Ref. [4].

Another feature of the result (4.3) is that it depends on the same unknown coefficients as appear in the unnormalized current (3.4), namely the products $W_{45} \bar{c}_{\mathrm{SW}}$ and $W_{\mathrm{A}} \bar{c}_{\mathrm{A}}$. Thus if one were to do a fit to multiple physical quantities, incorporating the constraints implied by WChPT so as to improve the extrapolation to the continuum limit, the inclusion of the correctly normalized axial current would not increase the number of unknown parameters.

\section{Applications}

Expanding the renormalized axial current we obtain the tree-level result for the pion decay constant:

$$
f_{\pi, \text { tree }}=f\left(1+\frac{4}{f^{2}} \hat{a}\left(W_{45} \bar{c}_{\mathrm{SW}}+W_{\mathrm{A}} \bar{c}_{\mathrm{A}}\right)\left[2-z_{\mathrm{A}}(t)\right]\right) .
$$

This gives the form of the discretization errors expected in a lattice calculation of $f_{\pi}$. We stress once again that the result depends on the choice of renormalization condition [through $z_{\mathrm{A}}(t)$ ]. While expected, it is still striking to see such a dependence explicitly.

Various comments are in order. First, at this order in ChPT, the continuum result is simply $f$, which is correctly reproduced. Second, a consistency condition on the calculation is that the LECs can appear in physical quantities only in certain "physical" combinations [4], and $W_{45} \bar{c}_{\mathrm{SW}}+W_{\mathrm{A}} \bar{c}_{\mathrm{A}}$ is indeed such a combination. Third, the choice of underlying fermion action enters through the values of $\bar{c}_{\mathrm{SW}}$ and $\bar{c}_{\mathrm{A}}$, with the choice of the ultra-local current also affecting $\bar{c}_{\mathrm{A}}$. If only the action is 
improved then $\bar{c}_{\mathrm{SW}}=0$, and one still finds, as expected, an $\mathrm{O}(a)$ term because $\bar{c}_{\mathrm{A}} \neq 0$. If both action and current are improved, then one finds the expected absence of the $\mathrm{O}(a)$ term-independent of the choice of the details of the renormalization condition. Finally, we note that in comparison with previous results in the literature, Ref. [3] misses both $W_{\mathrm{A}}$ and $z_{\mathrm{A}}$ terms, while Ref. [4] effectively assumes that $z_{\mathrm{A}}=0$.

We have also done a one-loop calculation and find

$$
f_{\pi, 1-\text { loop }}=f\left(1+\frac{\hat{a}}{f^{2}} \tilde{W}_{\mathrm{A} 1}-\frac{1}{16 \pi^{2} f^{2}}\left[1+\frac{\hat{a}}{f^{2}} \tilde{W}_{\mathrm{A} 2}\right] M_{\pi}^{2} \ln \frac{M_{\pi}^{2}}{\mu^{2}}+\frac{8}{f^{2}} M_{\pi}^{2}\left[L_{45}+\frac{\hat{a}}{f^{2}} \tilde{W}_{\mathrm{A} 3}\right]\right)
$$

with $\tilde{W}_{\mathrm{A} 3}$ being a new unknown LEC while

$$
\tilde{W}_{\mathrm{A} 1}=4\left(W_{45} \bar{c}_{\mathrm{SW}}+W_{A} \bar{c}_{\mathrm{A}}\right)\left[2-z_{A}(t)\right] \quad \tilde{W}_{\mathrm{A} 2}=4\left(W_{45} \bar{c}_{\mathrm{SW}}+W_{A} \bar{c}_{\mathrm{A}}\right)\left[1-z_{A}(t)\right]
$$

Note that these two coefficients depend only on the physical combination $W_{45} \bar{c}_{\mathrm{SW}}+W_{A} \bar{c}_{\mathrm{A}}$ of LEC, as expected.

Our one-loop result correctly reproduces the continuum result of Gasser and Leutwyler [7] in the limit $a \rightarrow 0$. At non-zero lattice spacing, however, there appear additional terms of $\mathrm{O}(a)$, $\mathrm{O}\left(a M_{\pi}^{2}\right)$ and $\mathrm{O}\left(a M_{\pi}^{2} \ln M_{\pi}^{2}\right)$. Quite generally, the coefficient of the chiral logarithm receives a correction in form of the factor $\left[1+\hat{a} \tilde{W}_{\mathrm{A} 2} / f^{2}\right]$, so it not only depends on $f$ and the number of flavors, but also on the (non-universal) lattice artifacts encoded in the coefficient $\tilde{W}_{\mathrm{A} 2}$ [8]. An exception is the third case discussed in the previous section with $z_{A}(t)=1$. Here $\tilde{W}_{\mathrm{A} 2}=0$ and the chiral logarithm is free of $\mathrm{O}(a)$ corrections.

Note that the combination $L_{45}$ of Gasser-Leutwyler coefficients enters the one-loop result in form of the lattice spacing dependent combination $L_{45}^{\text {eff }}(a)=L_{45}+\hat{a} \tilde{W}_{\mathrm{A} 3} / f^{2}$. In order to obtain the physically interesting part $L_{45}$ one has to extrapolate to the continuum limit.

We close with a final remark on formula (5.2). For a consistent result to a given order one has to specify a power-counting scheme, and the literature usually distinguishes two regimes: (i) the GSM regime with $m \sim a \Lambda_{\mathrm{QCD}}^{2}$ and (ii) the LCE regime with $m \sim a^{2} \Lambda_{\mathrm{QCD}}^{3}$. Here GSM stands for generically small quark masses [4] and LCE for large cut-off effects [9]. Equation (5.2) is only a partial next-to-leading order (NLO) result for the LCE regime since we ignored the $\mathrm{O}\left(a^{2}\right)$ corrections in the effective action [10] and the effective currents. The NLO result for the GSM regime, however, is obtained from (5.2) by dropping the corrections proportional to $\tilde{W}_{\mathrm{A} 2}$ and $\tilde{W}_{\mathrm{A} 3}$, which are of next-to-next-to-leading order in this regime.

\section{Conclusions}

We have reconsidered the construction and matching of the vector and axial currents in WChPT. The explicit chiral symmetry breaking of Wilson fermions compels us to take two aspects into account which are not present in continuum ChPT:

1. The local lattice currents are not conserved, and in general they do not map onto the conserved currents in WChPT. In particular, the WChPT currents are not obtained by the Noether procedure, because the currents in the Symanzik theory have $\mathrm{O}(a)$ corrections which are not related to the effective action. 
2. The proper matching of the currents has to take into account the finite renormalization of the local lattice currents. The same renormalization conditions that have been employed for the lattice currents must be imposed on the effective currents in WChPT. Depending on the particular choice for the renormalization conditions the expressions for the renormalized currents differ by terms of $\mathrm{O}(a)$.

A direct consequence is that WChPT predictions for matrix elements of the currents also depend on the renormalization condition one has adopted. This is not a flaw but rather reflects the fact that the lattice data differs too depending on the particular condition one has chosen. What we find is that this dependence enters at $\mathrm{O}(a)$, but that it does not introduce any new LECs.

Our results emphasize the perhaps rather obvious general point that non-perturbative renormalization conditions generically introduce additional discretization errors. These must be (and in practice usually are being) accounted for when extrapolating to the continuum limit.

\section{Acknowledgments}

This work is supported in part by the Grants-in-Aid for Scientific Research from the Ministry of Education, Culture, Sports, Science and Technology (Nos. 20340047,20105001,20105003), by the Deutsche Forschungsgemeinschaft (SFB/TR 09) and by the U.S. Department of Energy under grant no. DE-FG02-96ER40956.

\section{References}

[1] M. Lüscher, S. Sint, R. Sommer and P. Weisz, Nucl. Phys. B 478, 365 (1996) [arXiv:hep-lat/9605038].

[2] S. R. Sharpe and R. L. Singleton, Phys. Rev. D 58, 074501 (1998) [arXiv:hep-lat/9804028].

[3] G. Rupak and N. Shoresh, Phys. Rev. D 66, 054503 (2002) [arXiv:hep-lat/0201019].

[4] S. R. Sharpe and J. M. S. Wu, Phys. Rev. D 71, 074501 (2005) [arXiv:hep-lat/0411021].

[5] S. Aoki, O. Bär and S. R. Sharpe, Phys. Rev. D 80, 014506 (2009) [arXiv:0905.0804 [hep-lat]].

[6] S. R. Sharpe, in “Perspectives in Lattice QCD,", ed. Y. Kuramashi, World Scientific (2008), arXiv:hep-lat/0607016.

[7] J. Gasser and H. Leutwyler, Ann. Phys. 158 (1984) 142.

[8] S. Aoki, Phys. Rev. D 68, 054508 (2003) [arXiv:hep-lat/0306027].

[9] S. Aoki, O. Bär and B. Biedermann, Phys. Rev. D 78, 114501 (2008) [arXiv:0806.4863 [hep-lat]].

[10] O. Bär, G. Rupak and N. Shoresh, Phys. Rev. D 70, 034508 (2004) [arXiv:hep-lat/0306021]. 